\title{
Assessment of atherosclerotic carotid plaque volume with multidetector computed tomography angiography
}

\author{
Thomas T. de Weert · Cécile de Monyé · Erik Meijering • \\ Ronald Booij · Wiro J. Niessen · Diederik W. J. Dippel · \\ Aad van der Lugt
}

Received: 7 September 2007 / Accepted: 19 March 2008/Published online: 29 March 2008

(C) The Author(s) 2008

\begin{abstract}
Purpose The amount of atherosclerotic plaque and its components (calcifications, fibrous tissue, and lipid core) could be better predictors of acute events than the now currently used degree of stenosis. Therefore, we evaluated a dedicated software tool for volume measurements of atherosclerotic carotid plaque and its components in multidetector computed tomography angiography (MDCTA) images. Materials and Methods Data acquisition was approved by the Institutional Review Board and all patients
\end{abstract}

Aad van der Lugt is recipient of a fellowship from the Netherlands Organization for Scientific Research (NWO-KF grant no. 907-00-122).

Electronic supplementary material The online version of this article (doi:10.1007/s10554-008-9309-1) contains supplementary material, which is available to authorized users.

T. T. de Weert · C. de Monyé · E. Meijering ·

R. Booij · W. J. Niessen · A. van der Lugt $(\square)$

Department of Radiology, Erasmus MC, University

Medical Center Rotterdam, Dr. Molewaterplein 40,

3015 GD Rotterdam, The Netherlands

e-mail: a.vanderlugt@erasmusmc.nl

E. Meijering · W. J. Niessen

Department of Medical Informatics, Erasmus MC, University Medical Center Rotterdam, Rotterdam,

The Netherlands

D. W. J. Dippel

Department of Neurology, Erasmus MC, University

Medical Center Rotterdam, Rotterdam, The Netherlands gave written informed consent. MDCTA images of 56 carotid arteries were analyzed by three observers. Plaque volumes were assessed by manual drawing of the outer vessel contour. The luminal boundary was determined based on a Hounsfield-Unit (HU) threshold. The contribution of different components was measured by the number of voxels within defined ranges of HU-values (calcification $>130 \mathrm{HU}$, fibrous tissue 60-130 HU, lipid core $<60 \mathrm{HU}$ ). Interobserver variability (IOV) was assessed. Results Plaque volume was $1,259 \pm 621 \mathrm{~mm}^{3}$. The calcified, fibrous and lipid volumes were $238 \pm 252 \mathrm{~mm}^{3}, 647 \pm 277 \mathrm{~mm}^{3}$ and $376 \pm 283 \mathrm{~mm}^{3}$, respectively. IOV was moderate with interclass correlation coefficients (ICC) ranging from 0.76 to 0.99 and coefficients of variation (COV) ranging from $3 \%$ to $47 \%$. Conclusion Atherosclerotic carotid plaque volume and plaque component volumes can be assessed with MDCTA with a reasonable observer variability.

\section{Introduction}

It is well known that the severity of stenosis is an unreliable estimation of the amount of atherosclerotic plaque. In case of carotid atherosclerotic disease, this is both related to the carotid bulb, in which atherosclerotic plaque accumulates before it compromises the lumen, and to positive remodeling, the phenomenon that an artery may or may not enlarge in response to plaque accumulation [1]. Furthermore, it 
is current opinion that atherosclerotic plaque rupture plays an important role in acute events, like transient ischemic accidents (TIA) and minor stroke [2]. Rupture-prone plaques have specific morphological features: the most frequently seen vulnerable plaque type has a large lipid-rich core with a thin fibrous cap [2] and has proved to be an independent predictor of ischemic cerebrovascular events [3].

It is therefore hypothesized that the amount of atherosclerotic plaque and its components (calcifications, fibrous tissue, and lipid core) could be better predictors of acute events than the now clinically used degree of stenosis, and may be useful in the selection of patients who could benefit from therapeutic intervention.

Computed tomography angiography (CTA) has been established as an accurate modality to assess the presence of carotid atherosclerotic plaque and grade the severity of stenosis [4]. A recent in vitro and in vivo study showed that quantification of the area (two dimensional) of atherosclerotic carotid plaque and its components is possible in axial thin section multidetector computed tomography angiography (MDCTA) images, in good correlation $\left(R^{2}>0.73\right)$ with histology $[5,6]$. Further developments in the quantification software now enable to quantify the volume of atherosclerotic plaque and the volume of different plaque components (three dimensional).

The aim of this study was to evaluate this software tool for atherosclerotic plaque and plaque component volume measurements in MDCTA images of the carotid artery and to assess the observer variability of these measurements.

\section{Materials and methods}

Subjects

Twenty patients with a 0-29\% stenosis grade (based on NASCET [7] criteria) and twelve patients for each of the three other stenosis grades (30-49\%; 50-69\% and 70-99\%) at the symptomatic side were retrieved at random from a database $(n=421)$ of MDCTA examinations of patients with transient ischemic attack or minor stroke. In all 56 patients MDCTA had been performed as part of a research protocol that was approved by the Institutional Review Board and for which all patients had given written informed consent.
Scanning and image reconstruction

Scanning was performed on a 16-slice MDCT scanner (Siemens, Sensation 16, Erlangen, Germany) with a standardized protocol $(120 \mathrm{kVp}, 180 \mathrm{mAs}$, collimation $16 \times 0.75 \mathrm{~mm}$, table feed $12 \mathrm{~mm} /$ rotation, pitch 1) [8]. All patients received $80 \mathrm{ml}$ contrast material $(320 \mathrm{mg} / \mathrm{ml})$, followed by $40 \mathrm{ml}$ saline, both with an injection rate of $4 \mathrm{ml} / \mathrm{s}$ [9]. The radiation dose was $2.6 \mathrm{mSv}$.

Image reconstructions were made with field of view $120 \mathrm{~mm}$, matrix size $512 \times 512$ (yielding interpolated pixels of $0.2 \times 0.2 \mathrm{~mm}$, real in-plane resolution is $0.6 \times 0.6 \mathrm{~mm}$ ), slice thickness $1.0 \mathrm{~mm}$, increment $0.6 \mathrm{~mm}$ and with an intermediate reconstruction algorithm (B46: heart-view sharp) [6].

\section{Quantification and characterization}

Three observers independently assessed the presence of an atherosclerotic lesion, the length of the atherosclerotic lesion, the location of the bifurcation, lumen attenuation, and plaque volume and plaque component volumes. One of the observers assessed after 4 months for a second time the volumes in a subset of patients (half the population per stenosis degree, randomly chosen).

The criterion used for the presence of an atherosclerotic lesion was: the presence of a calcification and/or thickening of the vessel wall. The length of the atherosclerotic lesion was defined as the distance between the first (most proximal) image and the last (most distal) image on which the atherosclerotic lesion was present. The location of the bifurcation was defined as the first image with two separate lumina. Lumen attenuation was measured in the most proximal and distal image with atherosclerosis, and the mean lumen attenuation was calculated.

Plaque and plaque component areas were measured with a polymeasure plug-in developed by one of the co-authors (E.M.) for the freely available software package ImageJ (Rasband, National Institute of Mental Health, Bethesda, USA). This plug-in made it possible to draw manually regions of interest (ROI) in consecutive axial MDCT images and to automatically calculate the total number of pixels and the number of pixels of different Hounsfield value (HV) ranges within these ROI (Fig. 1). The ROI was placed over the outer vessel wall contour and 


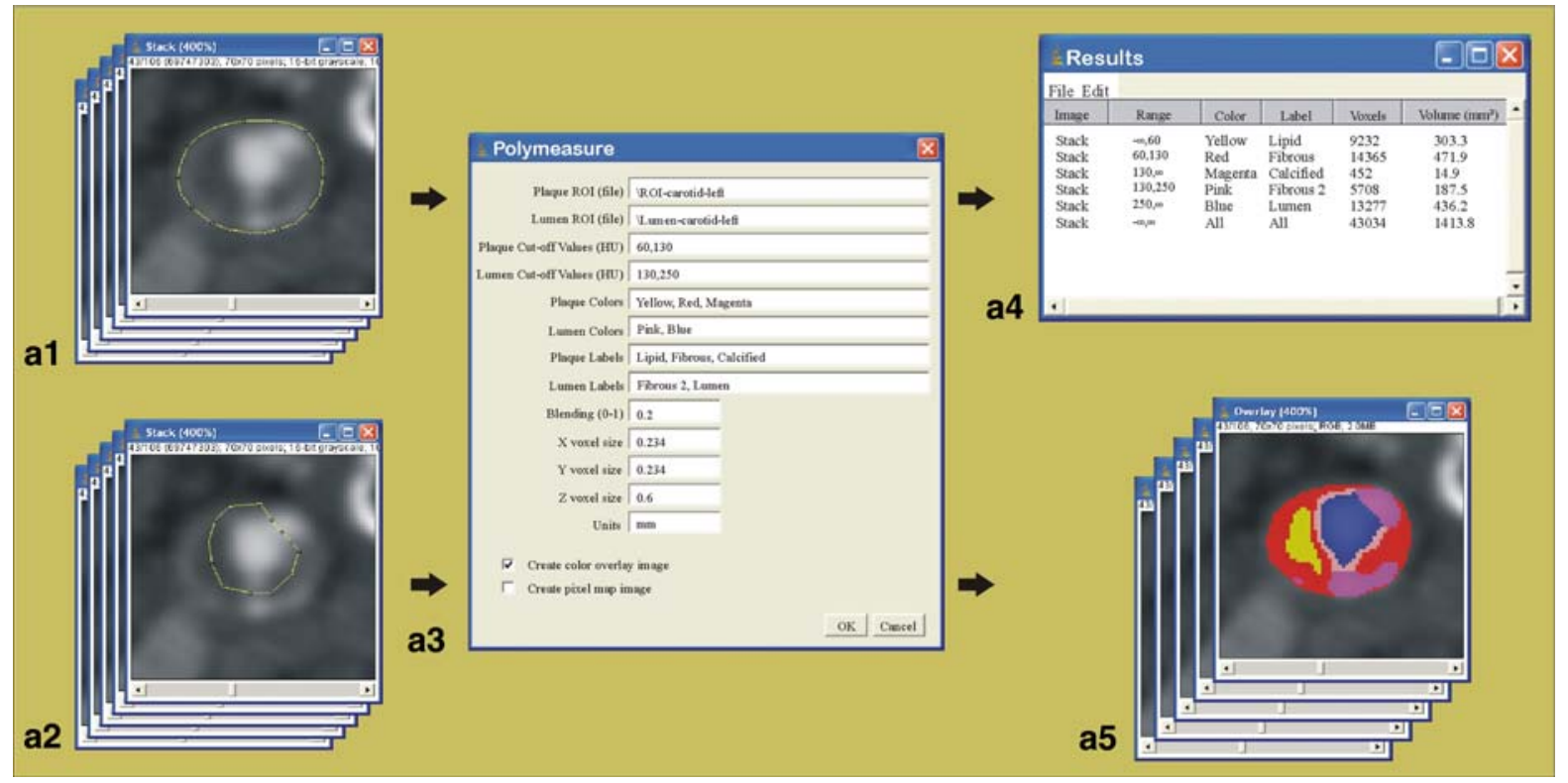

Fig. 1 Semi-automatic assessment of plaque component volumes in a stack of MDCTA images with the ImageJ plugin 'PolyMeasure'. (a1) This plug-in allows an observer to draw a region of interest (ROI) on consecutive axial MDCTA images. This ROI represents lumen area and atherosclerotic plaque area. (a2) To differentiate lumen area from the atherosclerotic plaque area and from calcified tissue, a second ROI is drawn. This second ROI should include the attenuated lumen area, but

therefore equals plaque area plus lumen area. The different $\mathrm{HV}$ ranges are considered to represent the different plaque components; calcification $>130 \mathrm{HU}$, fibrous tissue 60-130 HU and lipid core $<60 \mathrm{HU}$.

The cut-off value between calcifications and fibrous tissue was set at $130 \mathrm{HU}$; the value currently used for calcium scoring. The cut-off value between fibrous tissue and lipid core was set at $60 \mathrm{HU}$ as assessed in previous studies [5, 6]. The cut-off value between atherosclerotic plaque and lumen was adjusted for each patient and based on the fullwidth-half-maximum principle (mean lumen attenuation plus mean fibrous tissue attenuation $(\approx 88 \mathrm{HU})$ divided by two). To compensate for partial volume effects, related to a high lumen attenuation at the plaque-lumen border, the pixels around the lumen with a HV between $130 \mathrm{HU}$ and the adjusted cut-off value were considered to be fibrous tissue. To assess the border between lumen and atherosclerotic plaque it was necessary to draw a second ROI close to the lumen in each image. Normally, the lumen area was then automatically differentiated from atherosclerotic should not include any calcifications. (a3) After the input of the cut-off values that differentiate the specific plaque components and the lumen, the plaque components and the lumen can be labeled with a color. After the input of the voxel sizes, (a4) atherosclerotic plaque component volumes and lumen volume are automatically calculated, and (a5) color overlay images are produced on which the plaque components and the lumen have a specific color

plaque based on the adjusted cut-off value. But in those plaques in which calcifications bordered the lumen and the two dense structures merged with each other, lumen area and calcifications had to be separated by manual drawing.

The volumes were calculated as the product of the number of pixels, the pixel size and the increment.

\section{Analysis}

Firstly, the difference between observers in the assessment of the presence of an atherosclerotic lesion was assessed. Hereafter, consensus on the presence of an atherosclerotic lesion was achieved by a consensus reading between all three observers. Those image series that were appointed as having atherosclerosis were used for further analysis.

Secondly, differences between observers in the assessment of the length of the atherosclerotic lesion, the location of the bifurcation, lumen attenuation and plaque and plaque component volumes, were calculated. 
After assessment of the differences, a second consensus reading was held in order to achieve consensus about the length of the atherosclerotic lesion, the location of the bifurcation and lumen attenuation because these features influence the volume measurements. All observers had to adapt their assessments on grounds of this second consensus reading and hereafter plaque and plaque component volumes were calculated again and differences were evaluated. This recalculation provides observer variability measurements due to differences in the assessment of the outer vessel wall contour only.

In order to assess not only the variability in volume measurements, the overlap (similarity index) between the ROIs (outer contour) of the observers was assessed and expressed as a percentage $(2 \times$ pixels with overlap/(pixels ROI observer $\mathrm{A}+$ pixels ROI observer B) $\times 100 \%$ ).

Finally, the intra-observer differences in plaque and plaque component volume measurements were assessed.

\section{Statistics}

Continuous data were compared with a paired Student's $t$-test for which a $P$-value $<0.05$ was considered to indicate statistical significance.

Inter-observer differences in the assessment of the length of the atherosclerotic lesion, the location of the bifurcation and lumen attenuation were expressed as the mean \pm the standard deviation (SD), and as a coefficient of variation defined by the SD of the paired difference divided by the mean of the absolute values.

Inter- and intra-observer differences in plaque and plaque component volume measurements were presented with a mean $\pm \mathrm{SD}$, an interclass correlation coefficient (ICC) with $95 \%$ confidence interval and a coefficient of variation. The differences were also plotted against the mean value of the measurements (Bland-Altman plot).

\section{Results}

In the 36 patients with a stenosis degree of $30 \%$ or higher, all observers agreed on the presence of an atherosclerotic lesion. In the 20 patients with $0-29 \%$ stenosis, the presence of an atherosclerotic lesion was determined in 9 patients by three observers, in 1 patient by two observers, and in 1 patient by one observer. The consensus reading appointed 10 patients as having atherosclerosis, thus 46 patients were selected for further analysis.

The mean time used by an observer for the analysis of one artery was about $1 \mathrm{~h}$, almost entirely taken by the drawing of the outer vessel wall.

The assessment of the length of an atherosclerotic lesion was significantly different between observers, while the assessment of the location of the bifurcation and lumen attenuation was not significantly different ( $P>0.27$ and $P>0.49$, respectively).

The ICC was good for all volume measurements (range 0.53-0.96). The plaque and plaque component volumes measured by the three observers were significantly different and the coefficients of variation (COV) were moderate (range 13-58\%) (Table 1).

After the second consensus reading in which consensus was achieved about the length of the atherosclerotic lesion, the location of the bifurcation and lumen attenuation, the ICC improved and was excellent for all volume measurements (ICC > 0.80), except for the lipid core volume measurements $(\mathrm{ICC}=0.76(0.54-0.87))$, for which it was good (Table 2). The coefficients of variation between observers improved for all measurements: plaque volume (17-24\%), calcified volume (13-33\%), fibrous tissue volume (18-24\%), lipid core volume (37-47\%) and lumen volume (3-10\%) (Table 2). The COV between observers for the assessment of calcified volume percentage (15-26\%), fibrous volume percentage (10-15\%) and lipid core volume percentage (21-30\%) were also improved (Table 2).

From the Bland-Altman plots it can be observed that especially the differences between observers in plaque volume, fibrous tissue volume and lipid core volume measurements increase with a larger volume (see supplemental files, Fig. I).

The similarity indices $( \pm \mathrm{SD})$ between the ROIs assessed by observer 1 and 2, observer 1 and 3, and observer 2 and 3 were $91.3 \pm 3.0 \%, 90.9 \pm 2.9 \%$ and $90.0 \pm 4.5 \%$, respectively.

Intra-observer analysis was good with excellent ICC (all >0.94) and moderate to good COV for the assessment of plaque volume (11\%), calcified volume $(8 \%)$, fibrous tissue volume $(8 \%)$, lipid core volume (25\%) and lumen volume (5\%) (Table 3). The intraobserver COV for the assessment of calcified volume percentage $(10 \%)$, fibrous volume percentage $(6 \%)$ 
Table 1 Mean values, inter-observer differences, interclass correlation coefficients, coefficients of variation of atherosclerotic plaque features and volume measurements from 46 CTA datasets in which atherosclerosis was considered to be present

\begin{tabular}{lcccccc}
\hline & Mean $\pm \mathrm{SD}$ & Diff Obs 1-2 & Diff Obs 1-3 & Diff Obs 2-3 & ICC 95\% CI & CoV (range) (\%) \\
\hline Lesion length $(\mathrm{mm})$ & $27.3 \pm 10.6$ & $6.1 \pm 4.4^{*}$ & $1.7 \pm 5.2 *$ & $4.3 \pm 6.2^{*}$ & - & - \\
Image with bifurcation $\left(\mathrm{mm}^{2}\right)$ & - & $0.5 \pm 3.6$ & $0.3 \pm 1.7$ & $0.2 \pm 3.2$ & - & - \\
Lumen attenuation $(\mathrm{HU})$ & $217.4 \pm 36.9$ & $0.4 \pm 5.9$ & $0.7 \pm 3.9$ & $0.2 \pm 5.8$ & - & - \\
Plaque volume $\left(\mathrm{mm}^{3}\right)$ & $1,259 \pm 621$ & $167 \pm 278^{*}$ & $247 \pm 381 *$ & $80 \pm 446$ & $0.79(0.65-0.87)$ & $23-34$ \\
Calcified volume $\left(\mathrm{mm}^{3}\right)$ & $238 \pm 252$ & $37 \pm 68^{*}$ & $3 \pm 38$ & $40 \pm 82^{*}$ & $0.96(0.93-0.98)$ & $13-34$ \\
Fibrous volume $\left(\mathrm{mm}^{3}\right)$ & $647 \pm 277$ & $77 \pm 144^{*}$ & $92 \pm 191 *$ & $15 \pm 210$ & $0.76(0.63-0.85)$ & $23-31$ \\
Lipid volume $\left(\mathrm{mm}^{3}\right)$ & $376 \pm 283$ & $48 \pm 141^{*}$ & $153 \pm 204^{*}$ & $105 \pm 236^{*}$ & $0.70(0.51-0.82)$ & $42-58$ \\
Luminal volume $\left(\mathrm{mm}^{3}\right)$ & $879 \pm 459$ & $182 \pm 206^{*}$ & $51 \pm 226$ & $132 \pm 240^{*}$ & $0.84(0.71-0.91)$ & $23-27$ \\
Calcified volume $(\%)$ & $19 \pm 15$ & $1 \pm 9$ & $4 \pm 9 *$ & $2 \pm 6 *$ & $0.85(0.77-0.91)$ & $33-48$ \\
Fibrous volume $(\%)$ & $54 \pm 12$ & $2 \pm 14$ & $0 \pm 13$ & $2 \pm 7 *$ & $0.53(0.36-0.69)$ & $13-27$ \\
Lipid volume $(\%)$ & $27 \pm 13$ & $1 \pm 11$ & $3 \pm 11^{*}$ & $5 \pm 8 *$ & $0.68(0.53-0.80)$ & $30-44$
\end{tabular}

$*=t$-test $P$ value $<0.05 ;$ Diff $=$ Difference; $\mathrm{Obs}=$ Observer; $\mathrm{CoV}=$ Coefficient of variation; $\mathrm{ICC}=$ Interclass correlation; $\mathrm{CI}=$ Confidence interval

Table 2 Mean values, inter-observer differences, interclass correlation coefficients, coefficients of variation of volume measurements from 46 CTA datasets in which atherosclerosis was considered to be present and consensus was reached with regard to the lesion length, location of bifurcation and lumen attenuation

\begin{tabular}{lcccccc}
\hline & Mean $\pm \mathrm{SD}$ & Diff Obs 1-2 & Diff Obs 1-3 & Diff Obs 2-3 & ICC (95 \% CI) & CoV (range) (\%) \\
\hline Plaque volume $\left(\mathrm{mm}^{3}\right)$ & $1,223 \pm 606$ & $26 \pm 194$ & $218 \pm 255^{*}$ & $192 \pm 300^{*}$ & $0.88(0.75-0.94)$ & $17-24$ \\
Calcified volume $\left(\mathrm{mm}^{3}\right)$ & $235 \pm 250$ & $28 \pm 60^{*}$ & $4 \pm 28$ & $31 \pm 78^{*}$ & $0.97(0.95-0.98)$ & $13-33$ \\
Fibrous volume $\left(\mathrm{mm}^{3}\right)$ & $619 \pm 264$ & $21 \pm 72^{*}$ & $67 \pm 88^{*}$ & $87 \pm 106^{*}$ & $0.90(0.78-0.95)$ & $18-24$ \\
Lipid volume $\left(\mathrm{mm}^{3}\right)$ & $369 \pm 278$ & $20 \pm 120$ & $150 \pm 168^{*}$ & $130 \pm 186^{*}$ & $0.76(0.54-0.87)$ & $37-47$ \\
Luminal volume $\left(\mathrm{mm}^{3}\right)$ & $830 \pm 421$ & $13 \pm 76$ & $6 \pm 21$ & $18 \pm 86$ & $0.99(0.98-0.99)$ & $3-10$ \\
Calcified volume $(\%)$ & $18 \pm 15$ & $2 \pm 4^{*}$ & $2 \pm 3^{*}$ & $4 \pm 5^{*}$ & $0.95(0.89-0.98)$ & $15-26$ \\
Fibrous volume $(\%)$ & $54 \pm 12$ & $2 \pm 6$ & $3 \pm 5^{*}$ & $1 \pm 8$ & $0.84(0.76-0.91)$ & $10-15$ \\
Lipid volume $(\%)$ & $27 \pm 13$ & $0 \pm 7$ & $5 \pm 6^{*}$ & $5 \pm 8^{*}$ & $0.81(0.66-0.89)$ & $21-30$
\end{tabular}

$*=t$-test $P$ value $<0.05 ;$ Diff $=$ Difference; $O b s=$ Observer; $\mathrm{CoV}=$ Coefficient of variation; $\mathrm{ICC}=$ Interclass correlation; $\mathrm{CI}=$ Confidence interval

and lipid core volume percentage (14\%) were also good (Table 3).

The similarity index $( \pm \mathrm{SD})$ between the two series of independently assessed ROIs by observer 1 was $93.7 \pm 1.8 \%$.

\section{Discussion}

Non-invasive in vivo assessment of atherosclerotic plaque volume and the relative contribution of the different plaque components will have important clinical implications: it provides new and probably better parameters, together with the severity of stenosis, for cardiovascular risk assessment, and furthermore the natural history of atherosclerotic disease and the effect of pharmacological intervention can be studied [10]. MDCTA has extensively been used to assess the severity of luminal narrowing, and nowadays attention is increasingly paid to the potential role of MDCTA in qualitative and quantitative evaluation of the atherosclerotic plaque itself. Validation studies in which image-based plaque features are compared with histology, as well as assessment of observer variability, are necessary to establish the final role of MDCTA in qualitative and quantitative atherosclerotic plaque evaluation.

Until now a few coronary in vivo studies have compared the plaque volume assessed with MDCTA and intravascular ultrasound (IVUS). One study 
Table 3 Mean values, intra-observer differences, interclass correlation coefficients, coefficients of variation of volume measurements from 46 CTA datasets in which atherosclerosis was considered to be present and consensus was reached with regard to the lesion length, location of bifurcation and lumen attenuation

\begin{tabular}{|c|c|c|c|c|}
\hline & Mean \pm SD & Difference & ICC $(95 \% \mathrm{CI})$ & $\mathrm{CoV}(\%)$ \\
\hline Plaque volume $\left(\mathrm{mm}^{3}\right)$ & $1,098 \pm 459$ & $1 \pm 120$ & $0.97(0.93-0.99)$ & 11 \\
\hline Calcified volume $\left(\mathrm{mm}^{3}\right)$ & $218 \pm 186$ & $7 \pm 18$ & $0.99(0.99-1.00)$ & 8 \\
\hline Fibrous volume $\left(\mathrm{mm}^{3}\right)$ & $591 \pm 229$ & $10 \pm 49$ & $0.98(0.95-0.99)$ & 8 \\
\hline Lipid volume $\left(\mathrm{mm}^{3}\right)$ & $289 \pm 205$ & $2 \pm 72$ & $0.94(0.87-0.97)$ & 25 \\
\hline Luminal volume $\left(\mathrm{mm}^{3}\right)$ & $824 \pm 413$ & $14 \pm 40$ & $1.00(0.99-1.00)$ & 5 \\
\hline Calcified volume (\%) & $20 \pm 16$ & $1 \pm 2$ & $0.99(0.98-1.00)$ & 10 \\
\hline Fibrous volume (\%) & $56 \pm 12$ & $1 \pm 3$ & $0.96(0.91-0.98)$ & 6 \\
\hline Lipid volume $(\%)$ & $24 \pm 11$ & $0 \pm 3$ & $0.96(0.91-0.96)$ & 14 \\
\hline
\end{tabular}

$*=t$-test $P$ value $<0.05 ; \mathrm{ICC}=$ Interclass correlation; $\mathrm{CI}=$ Confidence interval; $\mathrm{CoV}=\mathrm{Coefficient}$ of variation

found a strong correlation $(r=0.8)$ and an underestimation of the coronary plaque volume assessed with MDCT compared to IVUS [11]. Another study found a moderate correlation $(r=0.55)$ and an overestimation of coronary plaque area assessed with MDCTA compared to IVUS [12]. The discrepancies between these studies might be explained by the results of a third study that found a strong correlation coefficient $\left(r^{2}=0.69\right)$ with an underestimation of mixed and noncalcified plaque volumes, and a trend to overestimate calcified plaque volumes with MDCTA. In addition, they reported a moderate reproducibility in the assessment of plaque volume, with a coefficient of variation of $37 \%$ [13].

An in vivo study [5] on carotid atherosclerotic plaques revealed a strong correlation between MDCTA and histology for the assessment of plaque area $\left(r^{2}=0.73\right)$; in addition, the inter- and intra-observer variability of plaque area measurements with MDCTA was reasonable with COV of $19 \%$ and $8 \%$, respectively.

To our knowledge, the present study is the first study that shows that in vivo quantification of the volume of atherosclerotic carotid plaque and its components is possible with MDCTA (Fig. 2). Inter-observer variability was moderate with ICC ranging from 0.53 to 0.96 and COV ranging from $13 \%$ to $58 \%$. To evaluate the inter-observer variability caused by the manual drawing of the contours we re-evaluated the data after consensus was reached with regard to the length of atherosclerotic disease, the location of the bifurcation and lumen attenuation, because all these features also influenced the volume measurements. This led to a decreased variability with ICC ranging from 0.76 to 0.99 and COV ranging from $3 \%$ to $47 \%$. Intra-observer variability was less with ICC ranging from 0.94 to 1.00 and $\mathrm{COV}$ ranging from $5 \%$ to $25 \%$.

The first problem which causes a variability in volume measurements is the differentiation between a normal vessel wall and a slightly thickened (diseased) vessel wall. In a number of cases, observers disagree with regard to the presence of atherosclerotic disease in carotid arteries with a stenosis of 0-29\%. In such cases, the assessed plaque volume in such patients will be very low; the measured plaque volume in the two arteries in which the observers disagree on the presence of atherosclerotic disease was 609 and $245 \mathrm{~mm}^{3}$, while the mean plaque volume of all patients was $1,259 \pm 621 \mathrm{~mm}^{3}$.

Furthermore, the difficulty in differentiation between a normal vessel wall and a slightly thickened (diseased) vessel wall, influences the assessment of the most proximal and distal image with atherosclerosis and thus the length of the atherosclerotic lesion. Because the plaque volume measurements include the original vessel wall, inclusion of additional images with normal vessel wall increases the amount of measured volumes considerably.

The second problem is the manual outlining of the outer border of the vessel wall. Some parts of the vessel wall can easily be differentiated from the surrounding tissue due to the low density of peri-arterial fat or the presence of calcifications at the outer border of the plaque. However, other parts have the same density as the peri- and paravertebral and sternocleidomastoid muscle, which are frequently positioned along the artery. The erroneous manual inclusion of peri-arterial fat in the ROI leads to the classification of this fat as lipid in the plaque. This inclusion will vary between 
Fig. 2 One $\mathrm{mm}$ multiplanar reformat (a) and $2 \mathrm{~mm}$ maximum intensity projection (b) in the sagittal plane depicts the carotid bifurcation with an atherosclerotic plaque. The startpoint (Im 1) and endpoint (Im 50) of atherosclerotic plaque volume assessment in this patient, and the position (C, D, E) of the three thin sliced $(0.75 \mathrm{~mm})$ axial MDCT images (c, d and e) of the internal carotid artery and their associated color overlay images $\left(\mathbf{c}^{\mathbf{1}}, \mathbf{d}^{\mathbf{1}}\right.$ and $\left.\mathbf{e}^{\mathbf{1}}\right)$ are indicated. A graphical representation of the absolute (f) and relative (g) volume measurements of lumen, calcifications, fibrous tissue, and lipid per MDCT image. The $x$-axis represents the consecutive MDCT images, the $y$-axis represents the volume. (h) A table with the total lumen, total calcified, total fibrous tissue and total lipid volume
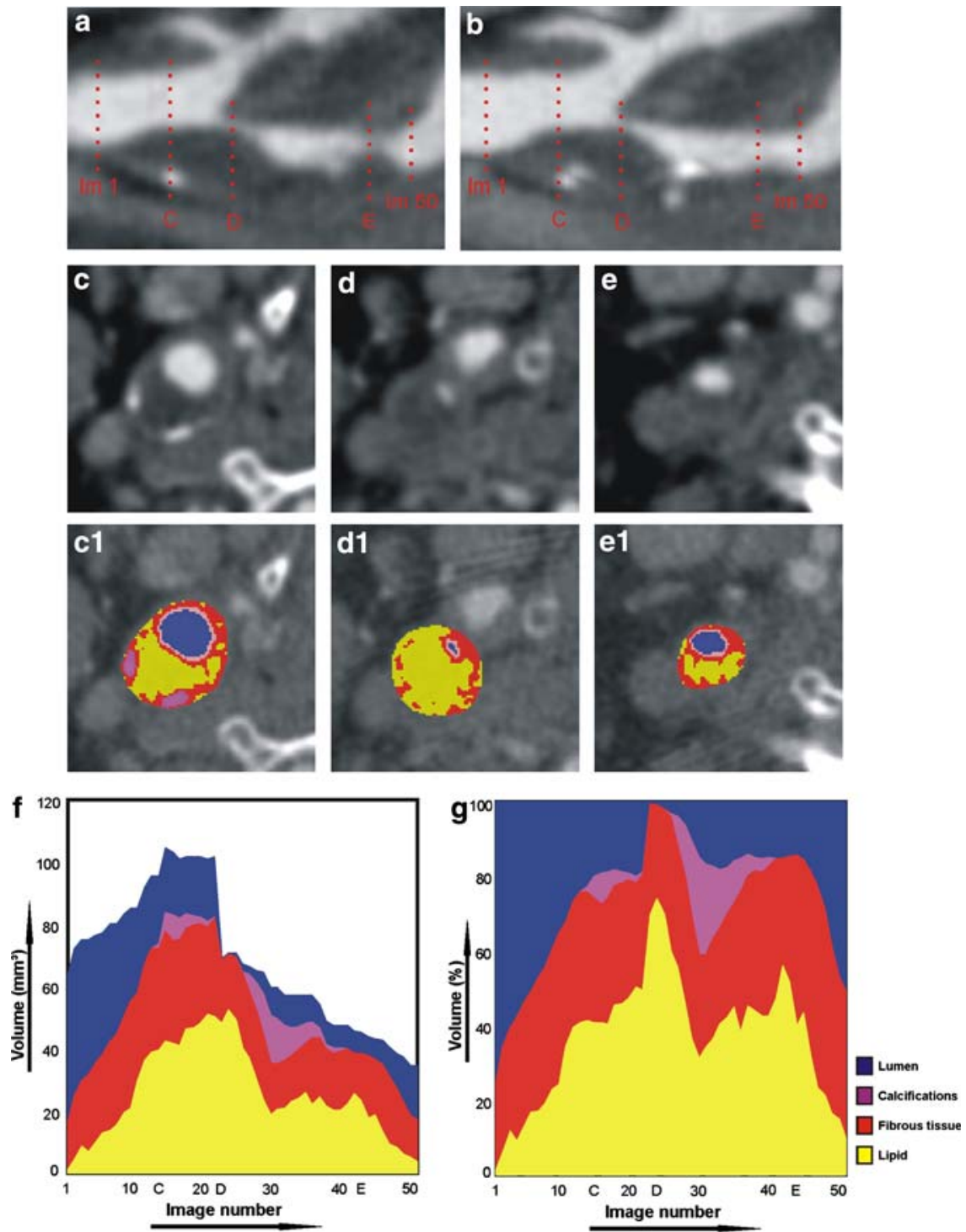

h

\begin{tabular}{|lrrlll|}
\hline Lumen & $527 \mathrm{~mm}^{3}$ & $25 \%$ & $\square$ Fibrous tissue & $701 \mathrm{~mm}^{2}$ & $34 \%$ \\
$\square$ Calcifications & $83 \mathrm{~mm}^{3}$ & $4 \%$ & $\square$ Lipid & $780 \mathrm{~mm}^{2}$ & $37 \%$ \\
\hline
\end{tabular}

the observers which explains the moderate ICC and the high observer variability in the assessment of lipid volume. The Bland-Altman plots confirm this by showing that the differences in lipid volume between observers 1 and 3 , and 2 and 3 depend on the size of the assessed volume, suggesting that observer 3 systematically draws a larger outer contour than the other observers and thus includes more peri-arterial low density tissues. An additional problem in the assessment of the outer border of the vessel wall is, that the size of calcifications is influenced by differences in window-level setting. Because these calcifications are often located at the border of an atherosclerotic plaque, different window-level settings between observers will influence the assessment of the outer vessel wall between observers, and thereby introduce variability in the assessment of plaque volume and calcified volume.

The third problem is the differentiation of contrastenhanced lumen from atherosclerotic plaque. In some plaque without calcifications at the inner border of the plaque the differentiation is automated and based on a threshold and the only variability is caused by a difference in the measurement of luminal attenuation, which was fortunately low. In case a calcification 
borders the lumen, a threshold based approach would merge the lumen with the calcification. In such cases manual drawing of the border between lumen and calcification was necessary which introduced a variability in plaque volume and calcium volume measurements.

We expect that improvements in the measurement software will improve the observer variability. Although in our method we assessed volumes, the analysis was performed in axial two-dimensional images. Evaluating the artery both in axial slices and using longitudinal reformats will provide more information on the borders of the vessel wall. This would enable a better continuation of transversal contours in adjacent slices. Also, highlighting specific parts of the vessel outer contour in axial images based on outer vessel contour assessment in longitudinal planes might be helpful. Finally, the differentiation between normal vessel wall and slightly thickened vessel wall can be based on wall thickness measurements, and the length of the atherosclerotic disease can be assessed more reproducibly.

Besides MDCTA, MRI has been used for noninvasive atherosclerotic carotid plaque characterization and quantification. Studies have shown that there is good agreement between in vivo MRI and histology for qualitative [14-16] and quantitative [17] assessment of plaque components, while observer reproducibility has shown to be good to excellent for plaque area [14-16, 18] and plaque component areas [19]. ICC for plaque area were $0.90-0.96$ and for lipid core $0.88-0.89$. The reproducibility of MRI-based plaque volume measurements has not been extensively studied. One study reported a coefficient of variation for an averaged (over 5 slices) plaque area of 3.5\% [20], while another study reported a coefficient of variation of $9.8 \%$ for plaque volume [20].

Until now carotid intima-media thickness (CIMT) is a validated endpoint in progression/regression studies. CIMT has shown to be an independent risk factor for future myocardial infarction and stroke risk [21, 22]. In addition, CIMT has been related to the presence of future carotid plaque [23]. Lifestyle changes [24] and statin therapy [25] has a beneficial effect on CIMT. Ultrasound assessment of CIMT is accurate when compared with histology [26, 27], and has a very good reproducibility (coefficient of variation $2.4-10.6 \%$ ) [28]. Interscan coefficient of variation is $5.6 \%$ [28], making it a potential valuable tool to evaluate the effectiveness of prevention therapy. Unfortunately, CIMT does not provide us with area and volumetric measurements of the plaque and ignores the presence of different plaque components. This makes CIMT unsuitable for the precise evaluation of pharmacological effects on the advanced atherosclerotic plaque.

The present study investigated the reproducibility of MDCTA-based atherosclerotic plaque volume measurements. It is a limitation that validation with histology has not been performed. Because we investigated a range of carotid artery stenoses, atherosclerotic specimens were not available in most of the patients. In the patients with a stenosis of more than $70 \%$ stent placement or surgery was performed. Previous studies [5, 6], however, have demonstrated a good correlation between area measurements with MDCTA and histology. A second limitation is the inclusion of the vessel wall (tunica media) in the plaque volume measurements. With MDCTA it is not possible to differentiate between the atherosclerotic plaque and the tunica media. This will lead to a systematic overestimation of plaque volume measurements. It is not expected that this overestimation will be a problem for serial evaluation or risk prediction.

\section{Conclusion}

In vivo assessment of atherosclerotic plaque and plaque component volumes in carotid arteries with MDCTA is feasible with a moderate reproducibility. A prospective longitudinal study which examines the relationship between cardiovascular risk factors, plaque and plaque component volumes and outcome may determine the value of MDCTA-based stroke risk predictors.

Acknowledgements This research was supported by Economic Affairs innovation grant IS044070 Automatic Diagnostic Vascular Analysis of CTA Examinations (ADVANCE)

Open Access This article is distributed under the terms of the Creative Commons Attribution Noncommercial License which permits any noncommercial use, distribution, and reproduction in any medium, provided the original author(s) and source are credited.

\section{References}

1. Glagov S et al (1987) Compensatory enlargement of human atherosclerotic coronary arteries. N Engl J Med 316:1371-1375 
2. Naghavi M et al (2003) From vulnerable plaque to vulnerable patient: a call for new definitions and risk assessment strategies: Part I. Circulation 108:1664-1672

3. Polak JF et al (1998) Hypoechoic plaque at US of the carotid artery: an independent risk factor for incident stroke in adults aged 65 years or older. Cardiovascular health study. Radiology 208:649-654

4. Koelemay MJ et al (2004) Systematic review of computed tomographic angiography for assessment of carotid artery disease. Stroke 35:2306-2312

5. de Weert TT et al (2006) In vivo characterization and quantification of atherosclerotic carotid plaque components with multidetector computed tomography and histopathological correlation. Arterioscler Thromb Vasc Biol 26:2366-2372

6. de Weert TT et al (2005) In vitro characterization of atherosclerotic carotid plaque with multidetector computed tomography and histopathological correlation. Eur Radiol 15:1906-1914

7. Beneficial effect of carotid endarterectomy in symptomatic patients with high-grade carotid stenosis (1991) North American symptomatic carotid endarterectomy trial collaborators. N Engl J Med 325:445-453

8. de Monye C et al (2006) Optimization of CT angiography of the carotid artery with a 16-MDCT scanner: craniocaudal scan direction reduces contrast material-related perivenous artifacts. AJR Am J Roentgenol 186:1737-1745

9. de Monye $\mathrm{C}$ et al (2005) Sixteen-detector row CT angiography of carotid arteries: comparison of different volumes of contrast material with and without a bolus chaser. Radiology 237:555-562

10. Pulido MA et al (2004) Imaging of atherosclerotic plaque. Int J Cardiovasc Imaging 20:553-559

11. Achenbach S et al (2004) Detection of calcified and noncalcified coronary atherosclerotic plaque by contrastenhanced, submillimeter multidetector spiral computed tomography: a segment-based comparison with intravascular ultrasound. Circulation 109:14-17

12. Moselewski F et al (2004) Comparison of measurement of cross-sectional coronary atherosclerotic plaque and vessel areas by 16-slice multidetector computed tomography versus intravascular ultrasound. Am J Cardiol 94:1294-1297

13. Leber AW et al (2006) Accuracy of 64-slice computed tomography to classify and quantify plaque volumes in the proximal coronary system: a comparative study using intravascular ultrasound. J Am Coll Cardiol 47:672-677

14. Cai $\mathbf{J}$ et al (2005) In vivo quantitative measurement of intact fibrous cap and lipid-rich necrotic core size in atherosclerotic carotid plaque: comparison of high-resolution, contrast-enhanced magnetic resonance imaging and histology. Circulation 112:3437-3444
15. Trivedi RA et al (2004) Multi-sequence in vivo MRI can quantify fibrous cap and lipid core components in human carotid atherosclerotic plaques. Eur J Vasc Endovasc Surg 28:207-213

16. Saam T et al (2005) Quantitative evaluation of carotid plaque composition by in vivo MRI. Arterioscler Thromb Vasc Biol 25:234-239

17. Yuan C et al (1998) Measurement of atherosclerotic carotid plaque size in vivo using high resolution magnetic resonance imaging. Circulation 98:2666-2671

18. Takaya $\mathrm{N}$ et al (2006) Intra- and interreader reproducibility of magnetic resonance imaging for quantifying the lipidrich necrotic core is improved with gadolinium contrast enhancement. J Magn Reson Imaging 24:203-210

19. Corti R et al (2001) Effects of lipid-lowering by simvastatin on human atherosclerotic lesions: a longitudinal study by high-resolution, noninvasive magnetic resonance imaging. Circulation 104:249-252

20. Adams GJ et al (2004) Tracking regression and progression of atherosclerosis in human carotid arteries using high-resolution magnetic resonance imaging. Magn Reson Imaging 22:1249-1258

21. O'Leary DH et al (1999) Carotid-artery intima and media thickness as a risk factor for myocardial infarction and stroke in older adults. Cardiovascular health study collaborative research group. N Engl J Med 340:14-22

22. Touboul PJ et al (2005) Carotid intima-media thickness, plaques, and Framingham risk score as independent determinants of stroke risk. Stroke 36:1741-1745

23. Zureik M et al (1999) Cross-sectional and 4-year longitudinal associations between brachial pulse pressure and common carotid intima-media thickness in a general population. The EVA study. Stroke 30:550-555

24. Markus RA et al (1997) Influence of lifestyle modification on atherosclerotic progression determined by ultrasonographic change in the common carotid intima-media thickness. Am J Clin Nutr 65:1000-1004

25. Probstfield JL et al (1995) Results of the primary outcome measure and clinical events from the asymptomatic carotid artery progression study. Am J Cardiol 76:47C-53C

26. Wong $\mathrm{M}$ et al (1993) Ultrasonic-pathological comparison of the human arterial wall. Verification of intima-media thickness. Arterioscler Thromb 13:482-486

27. Pignoli $P$ et al (1986) Intimal plus medial thickness of the arterial wall: a direct measurement with ultrasound imaging. Circulation 74:1399-1406

28. Sramek A et al (2000) Ultrasound assessment of atherosclerotic vessel wall changes: reproducibility of intimamedia thickness measurements in carotid and femoral arteries. Invest Radiol 35:699-706 